\title{
Concentração de metais no rio Doce em Mariana, Minas Gerais, Brasil
}

\author{
Marllon Santos de Carvalho ${ }^{a^{*}}$, Raner Moacir Moreirab ${ }^{b}$ Kátia Daniela Ribeiro ${ }^{b}$, Alex Magalhães de Almeidab \\ ${ }^{a}$ Ecos Engenharia, Formiga, Minas Gerais, 35570-000, Brasil. * marllonsantos05@yahoo.com.br \\ a Centro Universitário de Formiga, Formiga, Minas Gerais, 35570-000, Brasil..
}

Recebido: 16 julho 2017 / Aceito: 25 setembro 2017 / Publicado online: 27 setembro 2017

\begin{abstract}
Resumo
Esta pesquisa avaliou a concentração de metais nas águas do rio Doce por metais pesados após o rompimento da barragem do Fundão, distrito de Bento Rodrigues, em Mariana, Minas Gerais. Amostras de água foram coletadas numa nascente a montante do local do rompimento (P1), a montante de um dique de contenção da lama despejada (P2) e a jusante do dique de contenção (P3), sendo caracterizadas quanto aos teores de chumbo $(\mathrm{Pb})$, arsênio (As), níquel (Ni), cobre ( $\mathrm{Cu})$, alumínio (Al) e manganês (Mn) por espectrofotometria. As maiores concentrações dos metais foram observadas para o ponto P2, nas seguintes concentrações (em mg L-1): $\mathrm{Pb}(0,097)$; As $(0,911)$; $\mathrm{Ni}(1,078) ; \mathrm{Cu}(1,115) ; \mathrm{Al}(9,432)$ e $\mathrm{Mn}(1,638)$, sendo a concentração de cobre, nesse ponto, 86 vezes maior que o valor máximo permitido (VMP) pela legislação brasileira, para Classe III de qualidade das águas. No ponto $\mathrm{P} 1$, as concentrações de $\mathrm{Ni}\left(0,051 \mathrm{mg} \mathrm{L}^{-1}\right), \mathrm{Cu}\left(0,062 \mathrm{mg} \mathrm{L}^{-1}\right)$ e $\mathrm{Al}\left(0,465 \mathrm{mg} \mathrm{L}^{-1}\right)$ apresentaram-se 2,$0 ; 4,8$ e 2,3 vezes maiores que os VMP's, respectivamente, podendo-se relacionar esses resultados à natureza mineralógica da área. As elevadas concentrações desses metais na água do rio podem estar provavelmente relacionadas ao desastre ambiental de Mariana, Minas Gerais, com rejeitos de minas. Ações de políticas ambientais e de recuperação da área são essenciais para o reequilíbrio das características químicas desse ecossistema aquático.
\end{abstract}

Palavras-chave: Heavy metals, Mining, Water quality, Waste.

\section{Concentration of metals in the Doce river in Mariana, Minas Gerais, Brazil}

\begin{abstract}
This research evaluated the concentrations of metals of Doce River water after the rupture of Fundão Dam, located at Bento Rodrigues District, in Mariana City, Minas Gerais, Brazil. Water samples were collected at a source upstream of the rupture site (P1), upstream of a containment embankment of the dumped sludge (P2) and downstream of the containment embankment (P3). Concentrations of lead $(\mathrm{Pb})$, arsenic $(\mathrm{As})$, nickel $(\mathrm{Ni})$, copper $(\mathrm{Cu})$, aluminum $(\mathrm{Al})$ and manganese $(\mathrm{Mn})$ in the samples were determined by spectrophotometry. The highest concentrations were observed at point $\mathrm{P} 2$, the concentrations found were (em $\left.\mathrm{mg} \mathrm{L}^{-1}\right)$ : $\mathrm{Pb}(0,097)$; As $(0,911)^{1}$; $\mathrm{Ni}(1,078)$; $\mathrm{Cu}(1,115)$; Al $(9,432)$ e $\mathrm{Mn}(1,638)$, and copper concentration, at this point, was 86 times higher than the maximum permissible value (MPV) by Brazilian legislation, for Class III water quality . At point P1, concentrations of $\mathrm{Ni}\left(0.051 \mathrm{mg} \mathrm{L}^{-1}\right), \mathrm{Cu}\left(0.062 \mathrm{mg} \mathrm{L}^{-1}\right)$ and $\mathrm{Al}\left(0.465 \mathrm{mg} \mathrm{L}^{-1}\right)$ were $2.0 ; 4.8$ and 2.3 times greater than MPVs, respectively, and these results can be related to the local mineralogy. The high concentrations of these metals in river water are related to the environmental disaster of Mariana, Minas Gerais, with mine tailings. Environmental policy actions and area recovery are essential for rebalancing the chemical characteristics of this aquatic ecosystem.
\end{abstract}

Keywords: Heavy metals, Mining, Water quality, Waste.

\section{Introdução}

A extração mineral é um setor importante no desenvolvimento do país, sendo fundamental para vários segmentos industriais (Carvalho et al., 2012). Vários municípios brasileiros têm sua estrutura econômica baseada na exploração mineral, entretanto, a atividade gera uma grande quantidade de resíduos, que devem ser geridos adequadamente para minimizar os impactos ambientais dessa atividade.

A utilização de barragens de rejeitos em atividades de mineração é uma das formas de controle de resíduos que apresentam bons resultados quando bem executadas (Machado, 2007). Quando mal dimensionadas ou executadas, essas estruturas de contenção podem ser levadas à ruptura, tornando-se num agente de danos ambientais que, além de impactar na fauna, flora e na destruição da infraestrutura da sociedade próxima ao local, pode levar a perdas de vidas humanas (Duarte, 2008).

A cidade de Mariana, em Minas Gerias, faz parte do quadrilátero ferrífero, região de intensa atividade mineradora, onde, em novembro de 2015, ocorreu o rompimento da barragem de contenção de rejeitos do Fundão, contaminando o solo e as águas de uma extensa área da bacia do Rio Doce. 
E essa contaminação deu-se predominantemente pela incorporação de metais pesados que são, de um modo geral, muito nocivos aos seres vivos e ecossistemas.

Oliveira e Horn (2006) caracterizam os metais pesados como elementos traços, que são aqueles que, em condições naturais, se apresentam em concentrações muito baixas. $\mathrm{O}$ incremento desses elementos nos corpos hídricos tem impactos significativos na saúde humana e na biota aquática e tem ocorrido, principalmente, devido a atividades industriais e de mineração (Ribeiro et al., 2012). Como exemplo tem-se a contaminação por arsênio (As) que se tornou um problema em muitas partes do mundo como resultado do vazamento de rejeitos de mineração (Rodrigues e Malafaia, 2010).

Segundo Sousa (2009), A presença de chumbo $(\mathrm{Pb})$ nos corpos d'água não é comum, assim como o cobre $(\mathrm{Cu})$ e o alumínio ( $\mathrm{Al}$ ) geralmente aparecem em concentrações baixas. O manganês (Mn) atua, em baixas concentrações, em processos fisiológicos da fauna e flora aquática, sendo que o níquel (Ni) não tem função biológica conhecida e comumente é tóxico aos organismos. Ainda segundo o mesmo autor, altas concentrações desses elementos nos rios e córregos ocorrem em virtude de descargas industriais inadequadas e atividades de mineração.

Qualquer alteração no meio ambiente afeta o equilíbrio ecológico e, no caso do rompimento de uma barragem de rejeitos, as consequências são grandes e complexas. Uma vez que a água exerce o papel de transportadora de elementos pelo meio aquático e funciona como transmissora de contaminação para fauna, flora e seres humanos, torna-se necessário o monitoramento dos níveis de concentração dos metais pesados na água a fim de avaliar os riscos a eles associados (Oliveira e Horn, 2006).

Assim, esta pesquisa avaliou a contaminação das águas do rio Doce por metais pesados após o rompimento da barragem do Fundão, distrito de Bento Rodrigues, em Mariana, Minas Gerais.

\section{Material e Métodos}

Foram coletadas amostras de água no rio Doce, em três pontos localizados nas proximidades da região do distrito de Bento Rodrigues, município de Mariana, Minas Gerais (Tabela 1).

Tabela 1. Coordenadas geográficas dos pontos de amostragem no rio Doce.

\begin{tabular}{cccc}
\hline \multirow{2}{*}{ Ponto } & \multicolumn{3}{c}{ Coordenadas UTM* } \\
\cline { 2 - 4 } & $\mathrm{E}(\mathrm{m})$ & $\mathrm{N}(\mathrm{m})$ & Elevação $(\mathrm{m})$ \\
\hline P1 & 662.820 & 7.756 .661 & 673 \\
P2 & 664.527 & 7.760 .264 & 695 \\
P3 & 664.953 & 7.761 .310 & 661 \\
\hline "Datum WGS-84, fuso 23K & &
\end{tabular}

O ponto $\mathrm{P} 1$ corresponde a uma nascente situada numa área não afetada pela lama despejada pelo rompimento da barragem. A amostragem no ponto P2 foi realizada a montante de um dique construído para conter a lama despejada, tratando-se de um local contaminado. E a amostragem no ponto P3 foi realizada a jusante do dique construído para conter a lama, tratando-se também de um local contaminado.

As amostras de água foram coletadas e acondicionadas em recipientes de polietileno (PET) devidamente lavados com água destilada, sendo posteriormente identificadas, preservadas em caixa de isopor com gelo e transportadas para o laboratório, onde foram mantidas resfriadas até a determinação das concentrações dos metais pesados. Todas as amostras foram coletadas no dia 20 de agosto de 2016, no período compreendido entre 11:00 e 14:00 horas.

Os metais pesados determinados foram: chumbo, arsênio, cobre, níquel, alumínio e manganês. Para todas as amostras, as determinações foram feitas em triplicata através de espectrofotometria UV-VIS.

$\mathrm{Na}$ determinação dos teores de cobre, empregou-se inicialmente um estudo para obter o diagrama de Roozeboom através da titulação de pares utilizando os solventes água, metilisobutilcetona e etanol. O diagrama apresentou duas regiões de fases e, na região de fase única do diagrama, escolheu-se um ponto para o início da pesquisa, que correspondeu aos volumes $5,0 \mathrm{~mL}$ de água, $20,0 \mathrm{~mL}$ de etanol e 5,0 $\mathrm{mL}$ de metilisobutilcetona.

O solvente água contém o elemento metálico de interesse, o reagente metilisobutilcetona contém o reagente complexante e o etanol torna possível a mistura homogênea entre a fase aquosa e o solvente orgânico. Com os volumes definidos, foi preparada uma solução do reagente complexante, 8hidroxiquinolina (8HQ) em metilisobutilcetona (MIC) na concentração de $1,72 \times 10^{-2} \mathrm{~mol} \mathrm{~L}^{-1}$.

Posteriormente, utilizando a composição definida dos solventes e a solução de 8HQ em MIC, obteve-se os espectros para a solução contendo o complexo, que apresentou o comprimento de onda característico do composto coordenado. Utilizou-se um espectrofotômetro HATCH, com cubeta de quartzo de 1,0 cm de caminho óptico, esmerilhada na parte de contato e transparente nas faces de incidência do feixe de luz. As leituras foram realizadas entre 330 e $600 \mathrm{~nm}$, obtendo o valor máximo de absorvância $(\mathrm{ABS})$ em $\lambda \max =370 \mathrm{~nm}$. Com o comprimento de onda definido, prepararam-se várias soluções de cobre, entre $5 \mathrm{mg} \mathrm{L}^{-1}$ e $50 \mathrm{mg} \mathrm{L}^{-1}$ para a obtenção da curva de calibração. A curva de calibração elaborada apresentou as seguintes características: $\mathrm{ABS}=0,0063[\mathrm{Cu}]+$ 0,0031 , com um $R^{2}=0,9987$. Por fim, realizaram-se as análises de determinação das amostras de água.

$\mathrm{Na}$ determinação das concentrações dos demais metais, adotaram-se procedimentos semelhantes aos descritos anteriormente, variando-se os reagentes e componentes dos sistemas homogêneos, respeitando-se as particularidades de cada metal.

Dessa forma, na determinação dos teores de chumbo, utilizou-se como meio reacional um sistema homogêneo composto por: $2,0 \mathrm{~mL}$ de água, $12,0 \mathrm{~mL}$ de etanol e $5,0 \mathrm{~mL}$ de clorofórmio. Como reagente complexante, utilizou-se o 1-(2piridilazo)-2-naftol preparado em clorofórmio. As leituras de absorvância foram feitas em $\lambda \max =562 \mathrm{~nm}$ (máximo de $\mathrm{ABS}$ do complexo). Para obtenção da curva de calibração, utilizaram-se soluções de chumbo nas concentrações de 0,1 a $1,0 \mathrm{mg} \cdot \mathrm{L}^{-1}$, obtendo-se a equação $\mathrm{ABS}=1,4316[\mathrm{~Pb}]+0,0344$, sendo o valor do $\mathrm{R}^{2}=0,9969$.

Para avaliar o teor de arsênio, utilizou-se uma mistura de solventes formada por: água-etanol-clorofórmio na proporção de 2,0 $\mathrm{mL}, 10,0 \mathrm{~mL}$ e $2,0 \mathrm{~mL}$, respectivamente. Foi empregado o reagente complexante ditizona e as leituras de absorvância foram efetuadas em $\lambda \max =500 \mathrm{~nm}$. A curva de 
calibração foi confeccionada entre as concentrações de 0,2 a 2,0 mg.. $\mathrm{L}^{-1}$ de arsênio, obtendo-se a equação $\mathrm{ABS}=0,855$ [As] $+0,0427$ com $\mathrm{R}^{2}=0,9957$.

Para o níquel, utilizou-se o reagente complexante dimetilglioxima e as leituras de absorvância foram realizadas em $\lambda \max =490 \mathrm{~nm}$. Para obtenção da curva de calibração, utilizaram-se soluções de níquel nas concentrações de 1,0 a $30,0 \mathrm{mg} \cdot \mathrm{L}^{-1}$, obtendo-se a equação $\mathrm{ABS}=0,0565[\mathrm{Ni}]+$ 0,0189 , sendo o $\mathrm{R}^{2}=0,9971$.

Na determinação dos teores de manganês, foi utilizado um sistema ternário homogêneo de solventes constituído por água-etanol-clorofórmio e o reagente 8-hidroxiquinolina foi utilizado como complexante. As leituras de ABS foram feitas em $\lambda \max =420 \mathrm{~nm}$ (máximo de absorvância do complexo). Para obtenção da curva de calibração, utilizaram-se soluções de manganês nas concentrações de 0 a 0,25 mg. $\mathrm{L}^{-1}$, obtendose a equação $\mathrm{ABS}=0,0061[\mathrm{Mn}]+0,004$, proporcionando um $\mathrm{R}^{2}=0,9992$.

$\mathrm{Na}$ determinação dos teores de alumínio, não foi utilizado um sistema ternário de solventes. Primeiramente, fez-se a solubilização do complexante alizarina em etanol, obtendo-se uma solução na concentração de $2,5 \times 10^{-3}$ mol.L ${ }^{-1}$. O comprimento de onda para as leituras foi de $\lambda \max =550 \mathrm{~nm}$ e a curva de calibração foi elaborada com concentrações do metal entre 0 e 7,5 mg. $\mathrm{L}^{-1}$, obtendo-se a equação $\mathrm{ABS}=$ $0,1349[\mathrm{Al}]+0,0079$, apresentando um $\mathrm{R}^{2}=0,9994$.

As soluções utilizadas nos experimentos foram preparadas com reagentes de grau analítico ou superior e sempre se empregou água deionizada.

A análise estatística dos dados consistiu na determinação das médias e desvios padrão, seguida de análise de variância, segundo um delineamento inteiramente casualizado, com a aplicação do teste de Scott-Knott $(\alpha=0,05)$ para comparação das médias. Utilizando-se os valores médios dos parâmetros determinados, foram realizadas ainda análises de correlação. As análises estatísticas dos dados foram realizadas utilizandose o software SISVAR versão 5.6 (Ferreira, 2014).

Os dados obtidos também foram confrontados com a legislação vigente. A esse respeito, preconizou-se que os valores legais para comparação seriam aqueles relativos à Classe III de qualidade conforme Resolução $\mathrm{n}^{\circ} 357$ do Conselho Nacional de Meio Ambiente - CONAMA, de 17 março de 2005.

\section{Resultados e Discussão}

A Tabela 2 apresenta as concentrações dos metais pesados nas amostras de água analisadas. As menores concentrações, para todos os metais analisados, correspondem ao ponto $\mathrm{P} 1$, o que já era esperado, visto que esse ponto está situado a montante do local do rompimento da barragem de rejeitos.

Todavia, observa-se que, no ponto $\mathrm{P} 1$, as concentrações de $\mathrm{Ni}, \mathrm{Cu}$ e $\mathrm{Al}$ apresentaram-se 2,0; 4,8 e 2,3 vezes maiores, respectivamente, que os VMP's estabelecidos pela Resolução CONAMA 357/05, o que se deve, provavelmente, à natureza mineralógica da área, onde ocorrem mineralizações sulfetadas associadas a depósitos auríferos que, uma vez expostos ao intemperismo, liberam elementos traço para as águas e solo da região (MENDES, 2012).

Nos pontos P2 e P3, todas as concentrações encontraramse acima dos VMP's, o que pode ser atribuído à contaminação das águas do rio Doce pela lama da barragem de rejeitos rompida. Todavia, as informações relacionadas ao desastre são ainda divergentes quanto à contaminação.

Tabela 2. Concentrações médias $\left(\mathrm{mg} \mathrm{L}^{-1}\right)$ e desvios padrão dos metais pesados analisados para as amostras de água coletadas no rio Doce, Mariana, Minas Gerais.

\begin{tabular}{ccccc}
\hline \multirow{2}{*}{ Metal } & \multicolumn{3}{c}{ Ponto de amostragem } & \multirow{2}{*}{ VMP* $^{*}$} \\
\cline { 2 - 4 } & $\mathrm{P} 1$ & $\mathrm{P} 2$ & $\mathrm{P} 3$ & \\
\hline $\mathrm{Pb}$ & $0,013 \pm 0,0^{\mathrm{aA}}$ & $0,097 \pm 0,01^{\mathrm{bA}}$ & $0,089 \pm 0,00^{\mathrm{bA}}$ & 0,033 \\
$\mathrm{As}$ & $0,012 \pm 0,00^{\mathrm{aA}}$ & $0,911 \pm 0,01^{\mathrm{cB}}$ & $0,736 \pm 0,02^{\mathrm{bB}}$ & 0,033 \\
$\mathrm{Ni}$ & $0,051 \pm 0,01^{\mathrm{aB}}$ & $1,078 \pm 0,04^{\mathrm{bC}}$ & $1,056 \pm 0,02^{\mathrm{bC}}$ & 0,025 \\
$\mathrm{Cu}$ & $0,062 \pm 0,03^{\mathrm{aB}}$ & $1,115 \pm 0,02^{\mathrm{bC}}$ & $1,427 \pm 0,04^{\mathrm{cC}}$ & 0,013 \\
$\mathrm{Al}$ & $0,465 \pm 0,01^{\mathrm{aC}}$ & $9,432 \pm 0,09^{\mathrm{cE}}$ & $8,914 \pm 0,07^{\mathrm{bD}}$ & 0,2 \\
$\mathrm{Mn}$ & $0,041 \pm 0,02^{\mathrm{aB}}$ & $1,638 \pm 0,17^{\mathrm{bD}}$ & $0,655 \pm 0,58^{\mathrm{aB}}$ & 0,5 \\
\hline
\end{tabular}

Médias seguidas pela mesma letra minúscula nas linhas e pela mesma letra maiúscula nas colunas não diferem entre si pelo teste de Scott-Knott a 5\% de significância. $*$ VMP $=$ Valor máximo permitido pela Resolução CONAMA 357/05 para Classe III de qualidade das águas.

O estudo realizado pelo Grupo Independente para Avaliação do Impacto Ambiental (GIAIA, 2015), constatou níveis elevados de manganês, arsênio e chumbo na água do rio Doce. Enquanto que a avaliação de sedimentos e água realizada pelo Serviço Geológico do Brasil - CPRM (Brasil, 2015) não detectou metais dissolvidos na água que caracterizassem uma contaminação causada pelo desastre, visto que as concentrações de metais pesados na água foram compatíveis com registros anteriores.

Dos metais analisados, o $\mathrm{Pb}$ foi o que apresentou as menores concentrações em todos os pontos analisados. Porém, sua concentração nos pontos P2 e P3 foi 2,9 e 2,7 vezes, respectivamente, maior que o VMP estabelecido para Classe III de qualidade das águas. Esse resultado remete à necessidade, no caso do uso das águas do rio Doce para abastecimento humano, de um tratamento avançado, como a flotação iônica, que tende a elevar o custo do processo de tratamento.

Por ser um elemento bioacumulativo, o consumo a longo prazo de água com elevados teores de chumbo pode causar danos ao ser humano, como irritabilidade, tumores e danos neurológicos (Guimarães, 2013).

Os valores observados para o arsênio apresentaram comportamento semelhante aos do chumbo, porém, no caso dos pontos P2 e P3, suas concentrações ficaram bem mais acima do VMP. Isso é preocupante considerando-se que o arsênio é bioacumulativo e que não se sabe por quantos anos este elemento ficará presente nas águas do rio, o que poderá comprometer o ecossistema local por um longo período de tempo.

Rhodes (2010), analisando os sedimentos do rio Gualaxo do Norte, situado também no município de Mariana, Minas Gerais, verificaram elevadas concentrações de As $(8,6$ - 82,4 mg. $\mathrm{kg}^{-1}$ ) ocasionadas pela atividade garimpeira. $\mathrm{O}$ elevado teor de As presente nos sedimentos da região não implica necessariamente em sua disponibilização para o meio aquoso, mas pode ser uma das fontes desse elemento aos corpos d'água. 
Os metais $\mathrm{Ni}$ e $\mathrm{Cu}$ apresentaram, para um mesmo ponto amostral, as mesmas concentrações. No entanto, esses elementos foram os que apresentaram maior discrepância com relação aos VMP's.

Em quantidades elevadas, tanto o $\mathrm{Ni}$ quanto o $\mathrm{Cu}$ podem causar intoxicações, sendo que o $\mathrm{Cu}$, acima de $0,5 \mathrm{mg} \mathrm{L}^{-1}$, confere sabor desagradável à água, tornando-a implantável (Companhia de Tecnologia de Saneamento Ambiental CETESB, 2010).

Em relação aos peixes, as elevandas concentrações de $\mathrm{Ni}$ e $\mathrm{Cu}$ podem precipitar a secreção da mucosa produzida pelas brânquias dos peixes, obstruindo o espaço interlamelar e bloqueando o movimento normal dos filamentos branquiais, o que pode ter contribuído para a morte da fauna aquática do rio Doce pós o rompimento da barragem de rejeitos (Freitas et al, 2016).

O Al foi, entre os metais analisados, o que apresentou as maiores concentrações para todos os pontos de amostragem, verificando-se que, assim como ocorreu para o $\mathrm{Ni}$ e $\mathrm{Cu}$, seus valores encontram-se acima do VMP para todos os pontos.

Ao caracterizar os impactos do rompimento de uma barragem de rejeitos em Miraí, Minas Gerais, Rosado (2010) também verificou teores elevados de Al na água e acima dos VMP's, atribuindo essas altas concentrações ao acidente ocorrido.

Concentrações de alumínio nos corpos d'água superiores a 0,2 mg.L. $\mathrm{L}^{-1}$ podem gerar gosto desagradável à água (Di Bernardo e Paz, 2008). No Reino Unido, foi realizado um estudo epidemiológico relacionando o mal de Alzheimer à concentração de alumínio na água de consumo, indicando aumento de $50 \%$ na incidência da doença em pessoas que ingeriam água com concentrações superiores a $0,01 \mathrm{mg} \mathrm{L}^{-1}$. (Di Bernardo e Paz, 2008).

Quanto ao Mn, o valor médio para o ponto P1 enquadra-se até mesmo na Classe I de qualidade das águas (CONAMA, 2005), apresentando o melhor resultado observado para este ponto. Assim como ocorreu para os outros elementos analisados, nos pontos P2 e P3, os teores de Mn apresentaramse acima do VPM.

Maia (2017), analisando a qualidade das águas do rio Doce após o desastre de Mariana, Minas Gerais, também verificou elevado teor de Mn dissolvido em dois trechos do rio.

A ingestão de Mn em excesso pode causar distúrbios psiquiátricos como esquizofrenia e distúrbios neurológicos irreversíveis, similares a doença de Parkinson. (D’Oliveira et al., 2007).

Segundo Miranda (2015), o Serviço Autônomo de Água e Esgoto (SAAE) de Baixo Guandu analisou amostras coletadas no dia 18 de novembro de 2015, no lago da Usina de Mascarenhas, e o laudo apontou a presença de metais pesados muito acima dos parâmetros aceitáveis. As concentrações de zinco e manganês estavam 2,5 e 32 vezes maior que o referencial, e ferro e alumínio chegam às marcas impressionantes de 4,9 mil e 25 mil vezes as quantidades estabelecidas pelo CONAMA.

A Tabela 3 apresenta os valores máximos permitidos para os metais analisados estabelecidos por normas internacionais. A legislação brasileira é bem mais restritiva que a internacional. No entanto, de um modo geral, os teores de metais pesados observados para os pontos P2 e P3, quando comparados à legislação internacional, continuam acima dos valores permitidos, reforçando a preocupação quanto a qualidade das águas do rio Doce.

Tabela 3. Padrões internacionais de qualidade estabelecidos pela UPSH, WHO e CONAMA para águas de abastecimento.

\begin{tabular}{cccc}
\hline Elemento & UPSH standard & WHO standard & VMP \\
\hline $\mathrm{Pb}$ & 0,05 & 0,1 & 0,033 \\
$\mathrm{As}$ & 0,05 & 0,05 & 0,033 \\
$\mathrm{Ni}$ & - & - & 0,025 \\
$\mathrm{Cu}$ & 1,0 & 1,5 & 0,013 \\
$\mathrm{Al}$ & - & - & 0,2 \\
$\mathrm{Mn}$ & 0,05 & 0,5 & 0,5
\end{tabular}

Valores em $\mathrm{mg} \mathrm{L}^{-1}$. UPSH $=$ United States Public Health. WHO $=$ World Health Organization $. \mathrm{VMP}=$ Valor máximo permitido pela Resolução CONAMA 357/05 para Classe III de qualidade das águas. Fonte: Environmental Pollution (2017).

O ponto P1, entretanto, quando comparado aos padrões internacionais, encontra-se em condições adequadas para consumo.

A matriz de correlação dos dados determinados nesta pesquisa (Tabela 4), revelou que o Mn apresentou correlação moderada e significativa com o $\mathrm{Pb}$, As e Ni. Entretanto, não houve correlação significativa entre os teores de Mn com os teores de $\mathrm{Cu}$ e $\mathrm{Al}$ presentes nas águas do rio Doce. Carvalho et al. (2010), analisando as interações de metais pesados em solo, também encontrou correlação não significativa entre Mn e Al.

Tabela 4. Matriz de correlação entre os metais pesados determinados para o rio Doce, Mariana, Minas Gerais.

\begin{tabular}{ccccccc}
\hline Metal & $\mathrm{Pb}$ & $\mathrm{As}$ & $\mathrm{Ni}$ & $\mathrm{Cu}$ & $\mathrm{Al}$ & $\mathrm{Mn}$ \\
\hline $\mathrm{Pb}$ & 1,000 & & & & & \\
$\mathrm{As}$ & $0,995^{*}$ & 1,000 & & & & \\
$\mathrm{Ni}$ & $0,998^{*}$ & $0,986^{*}$ & 1,000 & & & \\
$\mathrm{Cu}$ & $0,953^{*}$ & $0,919^{*}$ & $0,972^{*}$ & 1,000 & & \\
$\mathrm{Al}$ & $0,999^{*}$ & $0,991^{*}$ & $0,999^{*}$ & $0,963 *$ & 1,000 & \\
$\mathrm{Mn}$ & $0,842^{*}$ & $0,891^{*}$ & $0,804^{*}$ & $0,640 \mathrm{~ns}$ & $0,823 \mathrm{~ns}$ & 1,000 \\
\hline
\end{tabular}

* significativo a 5\% de significância. ns: não significativo.

O Mn foi o único metal em que sua concentração, a jusante do local do desastre, retornou a valores estatisticamente iguais aos verificados na nascente (Tabela 2). Para os demais metais, suas concentrações permaneceram elevadas após o dique de contenção dos rejeitos despejados.

Isso indica um maior acúmulo desse metal no local do despejo da lama contaminada, fazendo com que seu comportamento, em termos de translocação pelo rio, fosse diferente dos demais, influenciando nos valores dos coeficientes de correlação determinados.

Ribeiro (2016), ao analisar o Rio Doce um ano após o desastre ambiental, constatou que o mesmo continua sem condições de uso e sem vida. A autora ainda relatou que, passados 12 meses, a contaminação não havia cessado e o arrasto de sedimentos pela bacia continuava. 
E apesar de muitos estudos não considerarem o rejeito despejado no rio Doce como tóxico, pois é formado basicamente por sílica, Lopes (2016) ressalta que o risco maior da contaminação não é para o ser humano, e sim para o meio ambiente. A lama trouxe prejuízos para todo o ecossistema local, impactando por anos seus rios, fauna, solo e até os moradores que têm contato direto com o rio e sedimentos da região.

\section{Conclusões}

As concentrações dos metais, no rio Doce, nos pontos amostrais investigados, se apresentam elevadas, ultrapassando os valores máximos permitidos pela legislação brasileira vigente, principalmente, Ações para recuperação da área são essenciais para o reequilíbrio das características químicas desse ecossistema aquático.

\section{Agradecimentos}

À Fundação de Ampara à Pesquisa do Estado de Minas Gerais (FAPEMIG) e ao Centro Universitário de Formiga (UNIFOR-MG).

\section{Referências}

Brasil. Serviço Geológico do Brasil - CPRM. 2015. Monitoramento especial da bacia do rio Doce: Relatório 02 - Geoquímica. Belo Horizonte, Minas Gerais.. Acesso em 24 set. 2017.

Carvalho, C.G.; Silva, J. M.; Curi, A.; Flores, J.C.C. 2012. A dependência da arrecadação do município de Ouro Preto do setor mineral. Revista Escola de Minas, 65(3), 385-392.

Carvalho, S.R.L. de; Vilas-Boas, G. da S.; Fadigas, F.S. 2010. Estimativa da concentração de metais pesados em solos dos tabuleiros do Recôncavo da Bahia. Cadernos de Geociências, 7(1).

Companhia de Tecnologia de Saneamento Ambiental - CETESB. 2010. Variáveis de qualidade das águas. Acesso em 24 set. 2017.

Conselho Nacional de Meio Ambiente - CONAMA. 2005. Resolução 357, de 17 de março de 2005. Publicada no DOU 053, de 18/03/2005. Acesso em 10 jul. 2017.

D’Oliveira, F.A.; Frank, A.A.; Soares, E. A. 2007. The influence of minerals on Parkinson's disease. Nutrire: Revista da Sociedade Brasileira de Alimentos e Nutrição, . Soc. Bras. Alim. Nutr.= Journal Brazilian of Society of Food and Nutrition, 32(1): 77-88.

Di Bernardo, L.; Paz, L.P.S. 2008. Seleção de tecnologias de tratamento de água. São Carlos, Editora LDIBE LTDA, 878p.

Duarte, A.P. 2008. Classificação das barragens de contenção de rejeitos de mineração e de resíduos industriais no estado de Minas Gerais em relação ao potencial de risco. Dissertação de Mestrado, Universidade Federal de Minas Gerais, Belo Horizonte, Minas Gerais. 130p.

Environmental Pollution. 2017. Water Pollution: Essay on Water Pollution (3817 Words). Acesso em 24 set. 2017.

Ferreira, D.F. 2014. Sisvar: a Guide for its Bootstrap procedures in multiple comparisons. Ciência e agrotecnologia [online]. 38(2): 109-112.

Freitas, C.M. de; SILVA, M.A. da; MENEZES, F.C. de. O desastre na barragem de mineração da Samarco: fratura exposta dos limites do Brasil na redução de risco de desastres. 2016. Ciência e Cultura. 68(3): 25-30.

Grupo Independente para Avaliação do Impacto Ambiental - GIAIA. 2015. Relatório parcial expedição rio Doce.

Guimarães, P.I.S. 2013. Estudo dos metais pesados na Amazônia: estudo de caso nas cidades de Novo Aripuanã, Barcelos e Manaus no estado do Amazonas - Brasil. Tese de Doutorado, Universidade Federal do Amazonas, Manaus, Amazonas. $203 \mathrm{f}$.

Lopes, L.M.N. 2016. O rompimento da barragem de Mariana e seus impactos socioambientais. Sinapse Múltipla, 5(1): 1-14.

Machado, W.G. de F. 2007. Monitoramento de barragens de contenção de rejeitos da mineração. Dissertação de Mestrado, Escola Politécnica da Universidade de São Paulo, São Paulo. 155 p.

Maia, F.F. 2017. Elementos traços em sedimentos e qualidade da água de rios afetados pelo rompimento da Barragem de Fundão, em Mariana, MG. Dissertação de Mestrado, Universidade Federal de Viçosa, Viçosa, Minas Gerais, $44 \mathrm{f}$.
Mendes, L.A. 2012. Avaliação da exposição da população de Passagem de Mariana (MG) aos elementos-traço, com ênfase ao arsênio. Dissertação de Mestrado, Universidade Federal de Ouro Preto, Ouro Preto, Minas Gerais. 147f.

Miranda, C. 2015. Laudos sobre qualidade da água do Rio Doce têm resultados divergentes. Acesso em 24 set. 2017.

Oliveira, M.R. de; Horn, A.H. 2006. Comparação da concentração de metais pesados nas águas do rio São Francisco em Três Marias, desde 1991 até hoje, relacionando a atuação da CMM-Três Marias. Revista Geonomos, 14(1, 2): 55-63.

Ribeiro, E.V. et al. 2012. Metais pesados e qualidade da agua do rio são Francisco no segmento entre Três Marias e Pirapora - MG: índice de contaminação. Revista Geonomos, 20(1).

Ribeiro, M. 2016. Rio Doce, um ano após tragédia, sem condições de uso ou sem vida. Acesso em 24 jul. 2017.

Rodrigues, A.S. de L.; Malafaia, G. 2010. A importância dos estudos sobre a contaminação por arsênio na saúde pública. SaBios - Revista de Saúde e Biologia, 5(2).

Rosado, V.B. 2010. Caracterização de sedimentos, solos e água e impactos do rompimento da Barragem São Francisco: o caso de Miraí, Minas Gerais. Tese de Doutorado, Universidade Federal de Viçosa, Viçosa, Minas Gerais. 103 f. Acesso em 24 set. 2017.

Sousa, J.K. C. 2009. Avaliação de impactos ambientais causados por metais traço em água, sedimento e material biológico na Baia de São Marcos, São Luís - Maranhão. Tese de Doutorado, Universidade Federal da Paraíba, João Pessoa, Paraíba. 110 f. 